\title{
An Enhanced Method of Contactless Charging of Railway Signaling Torch Light
}

\author{
K.Muthusamy \\ Department of ECE, Rathinam Technical Campus, Coimbatore, India \\ muthueee95@gmail.com \\ P.Rajesh \\ Department of ECE, Rathinam Technical Campus, Coimbatore, India \\ rajesh.ece@,rathinam.in \\ B.Gokulavasan \\ Department of ECE, Sri Eshwar College of Engineering, Coimbatore, India \\ gokul.cdab@gmail.com
}

Received: July 6, 2021. Revised: September 10, 2021. Accepted: September 15, 2021. Published: September 23, 2021.

\begin{abstract}
Wireless charging, also known as contactless charging (for shorter range), is a method of supplying energy to electrical devices by sending electricity via an air gap. Wireless charging methods have advanced recently, and commercial solutions have been developed, providing a potential option to overcome the energy bottleneck of typically portable battery-powered gadgets. Due to its simplicity and improved user experience, this technology is attracting a wide range of applications, from low-power gadgets to high-power electric cars. However, including wireless charging into the systems raises a number of difficult challenges in terms of implementation, scheduling, and power management. One such application is to convert the existing system of traditional battery powered railway signaling torchlight into a rechargeable type contactless charging system. This provides a better way of increasing the life time of the product and has better compactness. A rechargeable Li-ion battery must be installed in lieu of the old non-rechargeable battery. To achieve satisfactory efficiency, the magnetic resonance coupling technology of contactless charging can be utilized. Through a shorter air gap, electrical power is transmitted from the charging module (main coil) to the Torchlight (secondary coil). Overall, the present system's cost, size are reduced and lifetime is increased.
\end{abstract}

\section{Keywords}

NE555, LM7805, MC34063, Fly back Transformer, Contact less Charging, Magnetic Resonance Coupling

\section{INTRODUCTION}

Contactless charging is a method of transferring power from power source to load via an air gap. The technology makes life easier for users. Contactless charging has lately moved from concepts to standards, and it is used in commercial products, particularly mobile phones and portable devices. There are several advantages to using wireless charging.

1. It enhances user-friendliness by eliminating the trouble of connecting cords. The same charger may be used for devices of many manufacturers and models.

2. It improves product durability for contact-free devices. (Waterproof and dustproof).

3. It expands device adaptability, particularly for devices where replacing batteries or charging cords is costly, risky, or impossible.

Wireless charging allows for on-demand power, eliminating the problem of overcharging and lowering energy expenditures.

\section{EXISTING SYSTEM}

The existing system is a battery powered device. Operating at $6 \mathrm{~V}$ DC it has $41.5 \mathrm{~V}$ AA cells in series to satisfy the power requirements. The battery's output is utilized to power six multicolor LEDs through a microprocessor, allowing the user to choose the colour of the LED light. It has an average lifetime of 1.5-2 months based on an optimal use of $5 \mathrm{hrs}$ per day at standard operating conditions. It is used as a signaling device for trains coming into and going out of a railway station.

There are 3 different phases;

1. Green: It's an indication for the train to come into or to go out of the station.

2. Red: It acts as an indication to show the occupancy of tracks and platforms in a railway station

3. White: It is used to denote the moving state of a train in a particular station. For example: Intercity express trains won't halt in normal local stations. To convey that the train won't halt in a particular station and will keep moving, the white colour light is used.

The following disadvantages were observed in the existing system;

1). Non rechargeable

2). Low battery life 
3). Non compact

4). Heavy size

\section{PROPOSED SYSTEM}

The proposed system creates a contactless chargeable module for the railway signaling torch which has an input of $240 \mathrm{~V} 50 \mathrm{~Hz} \mathrm{AC}$ and an output of $6-8 \mathrm{~V}$ DC to charge a rechargeable $6 \mathrm{~V} \mathrm{Li-ion} \mathrm{battery.}$ Magnetic resonance coupling technique of contactless charging is used. An air gap of $2.4 \mathrm{~cm}$ is maintained on all sides (circular). The module is electrically compatible with the incoming power source and has an inbuilt circuit breaker unit that will turn off the incoming power supply to the module if the circuit fails. Electromagnetic interference has been extensively examined and shown to be limited within the air gap supplied. The strength of the magnetic field changes in inverse proportion to the cube of the distance from the coil's centre point. The module's charging current is $250-300 \mathrm{~mA}$. The frequency of the pulse signal is 15 $\mathrm{kHz}$. To minimize overheating, the coil's resonance frequency is adjusted to $32.5 \mathrm{kHz}$.

\section{Benefits:}

1. Connecting cables is no longer a problem, which improves user-friendliness. Using the same charger, you may charge a variety of brands and types of devices.

2. Allows for the design and manufacture of considerably smaller devices without the need for batteries.

3. It improves flexibility because replacing the batteries or connecting wires for charging is either too expensive, too dangerous, or too difficult.

4. On-demand power is provided to charging devices, making it more flexible and energy-efficient.

\section{WIRELESS CHARGING TECHNIQUES}

Three Major Techniques for Wireless Charging are 1. Inductive Coupling

2. Magnetic Resonance Coupling

3. Far Field Wireless Charging.

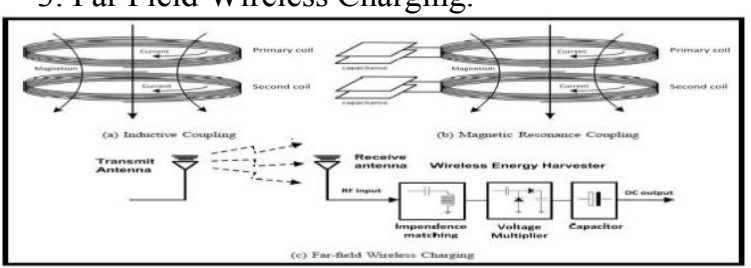

Fig 1: Wireless Charging Techniques

\section{BLOCK DIAGRAM OF PROPOSED SYSTEM}

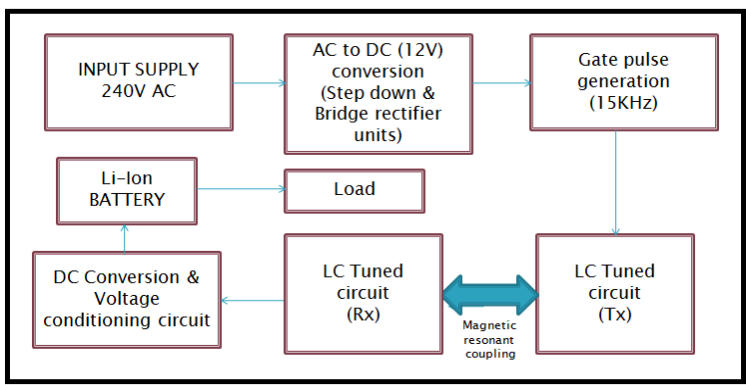

Fig 2: Block Diagram of Proposed System

The block diagram consists of 8 different blocks. They serve different purposes under each category.

Input supply: This is used to represent the incoming $240 \mathrm{~V} 50 \mathrm{~Hz}$ AC sine signal which is given as input to the charging module.

AC to DC conversion: This represents the normal step down process and converting the stepped down $\mathrm{AC}$ into a rectified filtered DC of suitable magnitude.

Gate pulse generation: This is used to perform the sampling process of converting a DC signal into a high frequency pulsating signal of suitable magnitude and frequency.

LC Tuned circuits:

The LC tuned circuits create an AC signal from the DC supply based on the pulsating signal obtained. The circuits are tuned to a particular frequency called the resonant frequency by choosing the appropriate value of inductance and capacitance. There are two different sections:

Transmitter: It is used to transmit the $\mathrm{AC}$ signal across the air gap through magnetic resonant coupling.

Receiver: It is used to receive the $\mathrm{AC}$ signal transmitted across the air gap through magnetic resonant coupling.

DC conversion and voltage conditioning circuit: The received AC Signal is converted back into DC again and the output value is obtained as per design.

Li-ion battery: The DC signal generated is given to a Li-ion battery for charging purposes.

Load: The output devices connected to the Battery receive their energy from the Li-ion battery.

\section{CIRCUIT DIAGRAM OF PROPOSED SYSTEM}

The circuit diagram can be split into 3 different categories

1. Source circuit: For providing the necessary input voltage to the transmitter section from the mains,

2. Transmitter circuit: For transmitting a AC signal across an air gap with the DC received as input 3. Receiver circuit: For receiving the AC signal across the air gap and convert it back into DC to charge a Li- 
ion battery.

\section{A. Source circuit:}

The source circuit is built around tiny series 2 TNY 266, a flyback transformer, EL817 opto coupler, and fast recovery and schottky diodes.

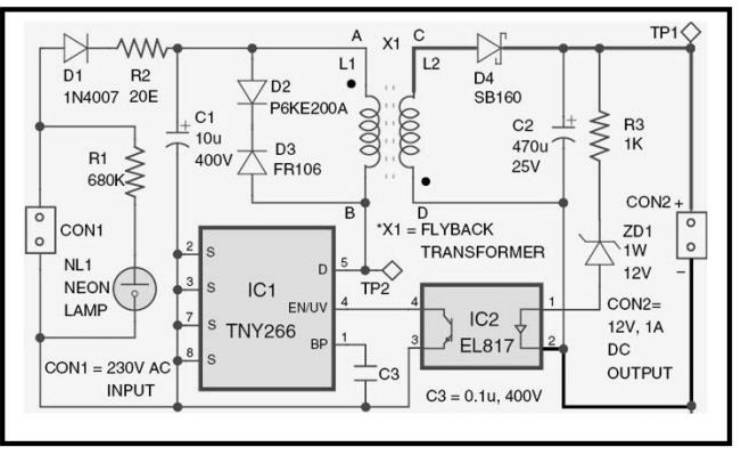

Fig 3: Source Circuit

\section{B. Transmitter circuit:}

Timer IC555, voltage regulator LM7805, transistor BC547, MOSFET, and LC tuned circuits are all used in the transmitter circuit.

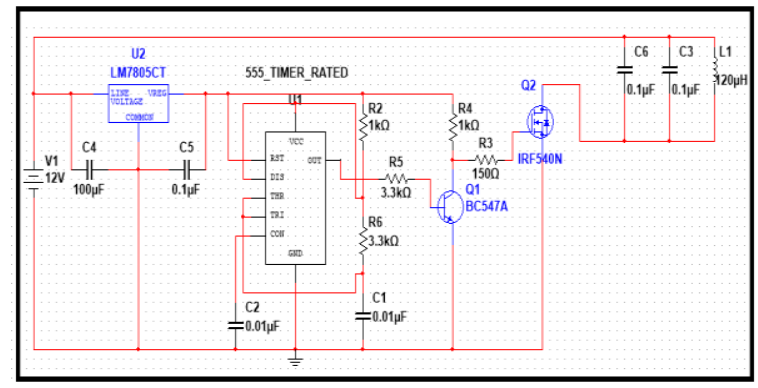

Fig 4: Transmitter Circuit

\section{Receiver Circuit:}

The receiver circuit consists of LC tuned circuits, a bridge rectifier, a filter, a Buck boost integrated circuit (MC34063) and current limiting resistors.

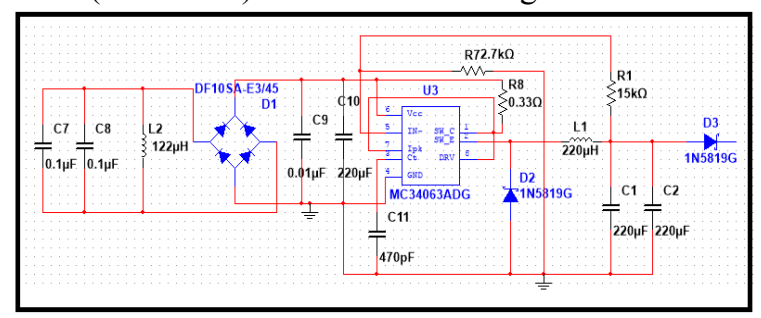

Fig 5: Receiver Circuit

\section{WORKING OF PROPOSED SYSTEM}

\section{A. Source circuit:}

A switched mode power supply (SMPS) can a chieve the same effects at a cheaper cost and with improved efficiency.

An SMPS is lighter and smaller than a convent ional power supply for a given output power. Because the frequency of operation is increased, a lower core cross-sectional area can be used. Furthermore, an ironcore transformer only works up to roughly $10 \mathrm{kHz}$, whereas a ferrite core is required for frequencies between 50 and $100 \mathrm{kHz}$.

Offline switcher with low power consumption (TNY266). The TNY266 chip, colloquially known as the "555" of SMPS, was used to create this SMPS. In a single package, this device contains a monolithic device, a $700 \mathrm{~V}$ power MOSFET, an oscillator, a highvoltage switching current source, current limiting, and thermal shutdown circuitry. As a result, a MOSFET with a Vdss of $650 \mathrm{~V}$ DC should be able to operate safely. Fortunately, TNY266 includes a MOSFET with these characteristics.

CON1 receives $230 \mathrm{~V} A C$ and is rectified by diode D1. When there is an input supply, the neon lamp (NL1) glows. The lamp's current is limited by the resistor R1. Coil L1's first terminal (A) is linked to the rectified output, the second terminal (B) of IC1 is connected to the drain of the integrated MOSFET. Diodes D2 and D3 are effectively snubbers, and they safeguard the MOSFET from exceeding 600V.

A flyback circuit is essentially a pair of inductors that are connected together. When a current is run through one inductor, it stores energy $\mathrm{E}=1 / 2\left({\mathrm{~L} . \mathrm{I}^{2}}^{2}\right.$, where ' $L$ ' represents inductance in henry and ' $\mathrm{I}$ ' represents current in amperes. This energy can then be extracted from the second inductor, which has a different volt-current ratio than the first. The energy storage and extraction process of the flyback is fascinating.

\section{B. Transmitter \& Receiver circuit:}

The Circuit Diagram here can deliver 250$300 \mathrm{~mA}$ at $7 \mathrm{~V}$. This circuit employs the inductive coupling approach. This circuit connects two LC tuned circuits at the same tuned frequency (tuned frequency of the transmitter must be equal to the tuned frequency of receiver). An tuned circuit generates and transmits magnetic fields, which are subsequently received by another tuned circuit.

The Asstable Multivibrator mode of the IC 555 Timer is used to create pulses. The IC 555 timer's output is connected to the transistor T1's base, which drives the MOSFET. The magnetic field oscillates when an LC tuned circuit is switched by a MOSFET.

The transmitter circuit is powered by 12 volts DC. The LM7805 is a series voltage regulator that provides a constant $+5 \mathrm{v}$, which is the transmitter circuit's operational voltage. The receiver circuit consists of LC tuned circuits, a bridge rectifier, a filter, a Buck boost integrated circuit (MC34063) and current limiting resistors. The LC tuned circuit, which comprises of L2, Capacitors C7 and C8, detects the transmitted magnetic field. The LC tuned circuit output is converted to DC using a Bridge Rectifier. 
The Buck boost regulator integrated circuit is coupled to the ripple-free dc voltage, which has been filtered by using LC filter. The filtered output is connected to the LED through the current limiting resistors R9 and R10.

\section{SIMULATION OF PROPOSED MODEL}

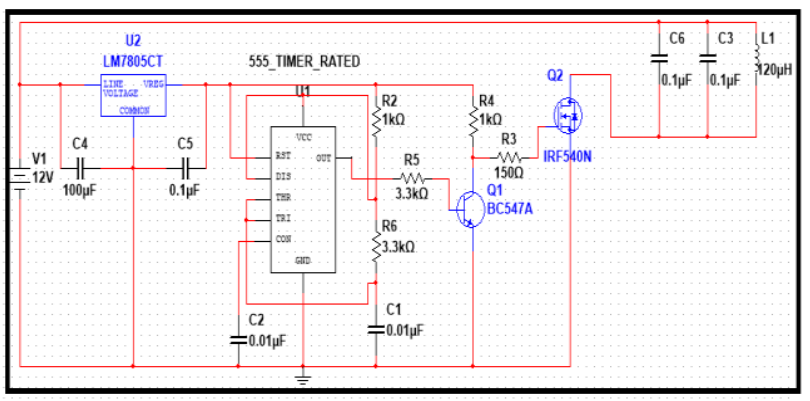

Fig 6: Simulation Circuit

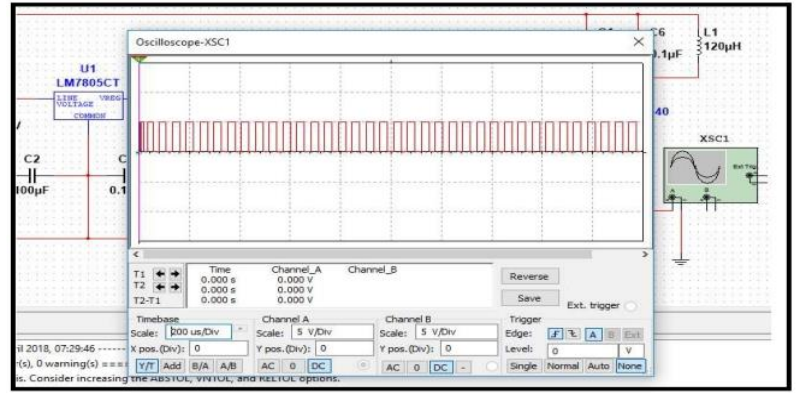

Fig 7: NE555 Timer Output

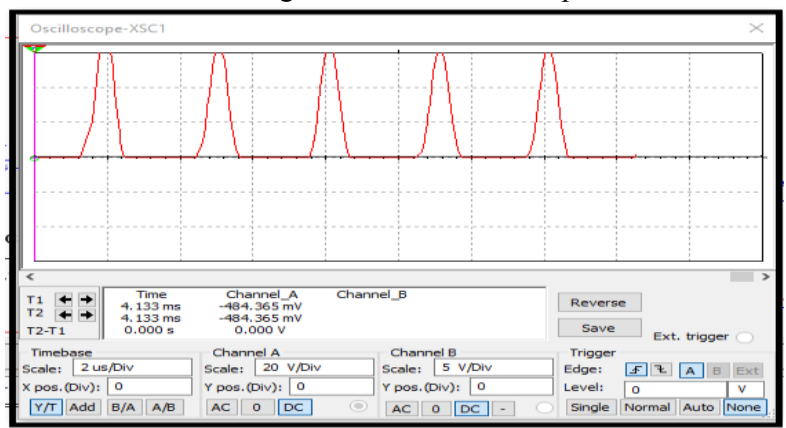

Fig 8: Drain Output Signal

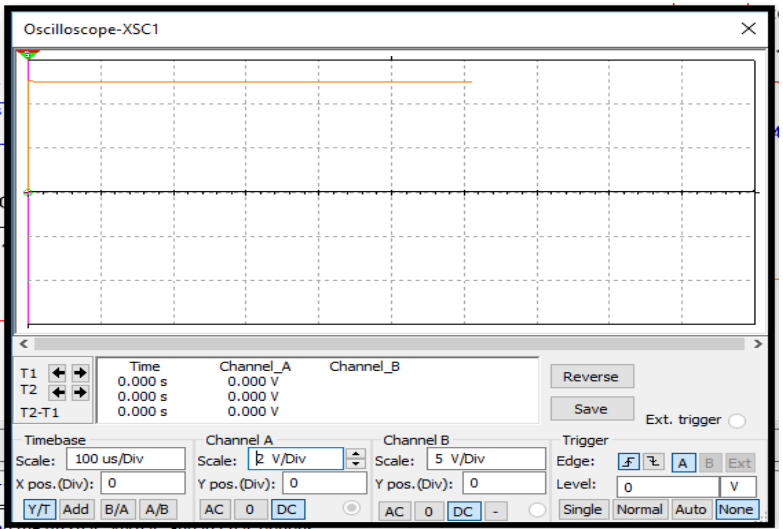

Fig 9: Generated DC Voltage

\section{WORKING MODEL OF PROPOSED SYSTEM}

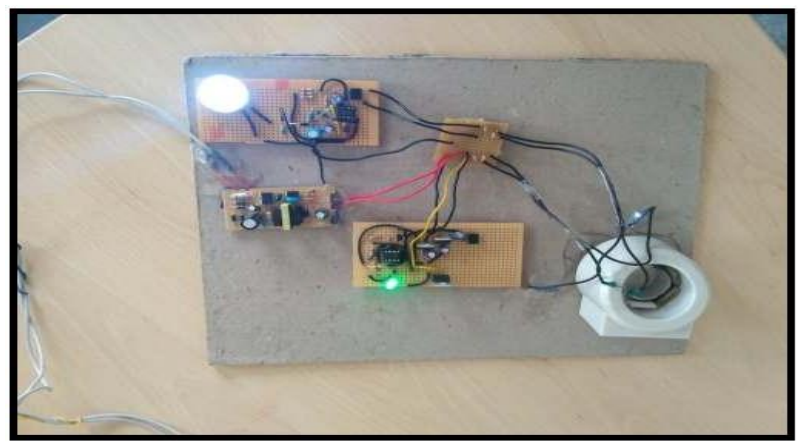

Fig 10. Circuit View of Proposed System (1)

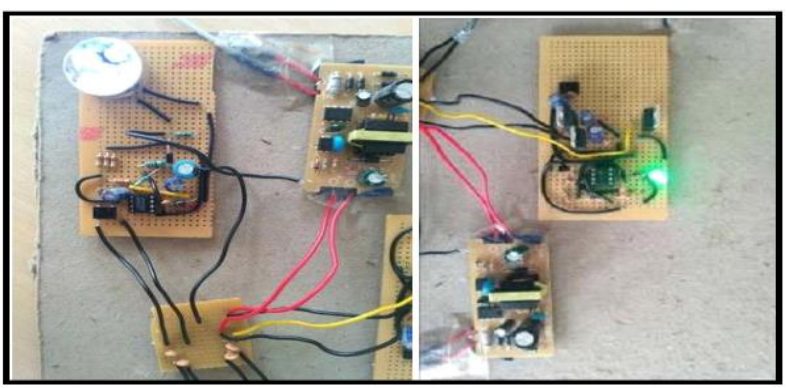

Fig 11: Circuit View of Proposed System (2)

\section{CONCLUSION}

This Proposed System offers an effective way to charge without any electrical contact the railway signaling torch which at present is Non Rechargeable. This System can contribute a lot of benefit to the railway management by increasing the life time of the Signaling Module and thereby preventing any mishaps or accidents that may occur due to malfunction of the signaling torch due to low life time at critical situations. The entire module is compact and is electrically compatible with the Indian Electrical Standard input voltage and frequency which means it has been charged over any plug point in India. Proper Electrical insulation and circuit breaker units have been installed in the module to prevent any possible electrical accidents. The electromagnetic Interference is minimum and decreases very rapidly after the specified air gap. The charging Current of the Module is sufficient enough to charge the rechargeable Li-ion battery in 1 to 1.5 Hours. The heat dissipation is optimal and power consumption is low.

\section{REFERENCES}

[1] Aakib J. Sayyad , N. P. Sarvade," Wireless Power Transmission for Charging Mobiles", International Journal of Engineering Trends and Technology (IJETT), Volume 12 Number 7 - Jun 2014.

[2] Dusit Niyato, Xiao Lu, Ping Wang, Dong In Kim, and Zhu Han "Wireless charger networking for mobile devices: fundamentals, standards, and applications" IEEE Communication surveys \& Tutorials, Volume 18, Issue 2 November 2015. 
[3] A.K.Shawney, "Design of Electrical Machines", Chapter- Transformer Design

[4] Design And Construction Of Wireless Charging System Using Inductive Coupling", International journal of scientific \& technology research, Volume 4, issue 06, June 2015.

[5] L Olvitz, D.Vinko, T.Swedek, "Wireless power transfer for mobile phone charging device", IEEE Xplore, MIPRO, 2012 Proceedings of the $35^{\text {th }}$ International Convention, May 2012.

[6] Tarique Salat, Shilpak Raich, Supriya Mahto and Shilpa Togarwar "A Wireless Battery Charger for Mobile Device", International Journal of Emerging Trends \& Technology in Computer science(IJETTCS), Volume 2, Issue 3, May - June 2013.

\section{Authors}

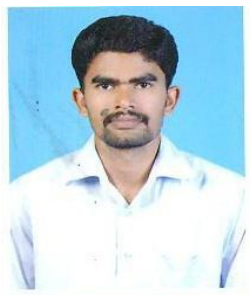

Mr. K. Muthusamy is pursuing his doctorate degree in Anna University, Chennai, India. His research interest Power Electronics, Wireless Communication,. He received M.E. in Power Electronics and Drives from Anna University, Chennai at 2014. He attended National and international conference in India, Published Papers in Reputed Journals. He is having Teaching Experience of 11 Years.

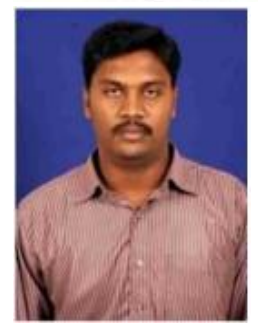

Mr. P. Rajesh received M.E. in VLSI design from Anna University, Chennai at 2012. His research interest VLSI Design, Wireless Communication. He is currently working as Assistant Professor at Rathinam Technical Campus, Coimbatore, India. He attended National and international conference in India, Published Papers in Reputed Journals. He is having Teaching Experience of 8 Years.

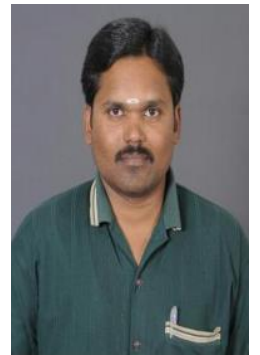

Mr. B. Gokulavasan is pursuing his doctorate degree in Karpagam Academy of Higher Education, Coimbatore, India. His research interests are Electrical Machines, Wireless Communication. He received M.E. in Applied Electronics from Anna University, Chennai at2008. He is currently working as Assistant Professor at Sri Eshwar College of Engineering, Coimbatore, India. He attended National and International conference in India and Published papers in Reputed journals. He is having Teaching Experience of 17 Years.

\section{Creative Commons Attribution License 4.0 (Attribution 4.0 International, CC BY 4.0)}

This article is published under the terms of the Creative Commons Attribution License 4.0

https://creativecommons.org/licenses/by/4.0/deed.en US 